Onkologie 1994;17:342-344

\title{
Inhalt, Vol. 17, No. 4, 1994
}

\section{Contents}

Übersichtsarbeiten

Die Familie der c-erẁB-Gene: Von der Grundlagenforschung zur Anwendung in der Klinischen Onkologie

Grunt, Th.W.,Huber,H 346

Die Rolle von Proteasen in Tumorinvasion und Metastasierung: Prognostische Bedeutung und therapeutische Herausforderung?

Wilhelm, O., Reuning, U, Jänicke, R, Schmitt, M., Graeff, H

Biochemische Modulation von Fluorpyrimidinen: Präklinische und klinische Untersuchungen

Mader, R. M, Rainer, H., Steger, G. G 368

Kurze Übersichten

Behandlung von Desmoidtumoren

Schroder, M., Queißer, W. 376

Primäres gastrointestinales Lymphom

Hünerbein, ML, Schlag, P. M384

Diagnostik und chirurgische Therapie des Magensarkoms

Lehnert, Th., Sinn, H.-P., Wolf, 0391

Therapie des Analkarzinoms

Witzigmann, H., Hehl, A. J., Meyer, F.M., Witte, J 398

Review Articles

The Family of c-erbB Genes: From Basic Research to Clinical Oncology

Grunt, Th.W.,Huber,H 346

The Role of Proteases in Tumor Invasion and Metastasis: Prognostic Impact and Therapeutical

Challenge?

Wilhelm, O., Reuning, U, Jänicke, F,, Schmitt, M., Graeff, H 358

Biochemical Modulation of Fluoropyrimidines: Preclinical and Clinical Studies

Mader, R. M, Rainer, H, Steger, G G 368

Short Reviews

Management of Desmoid Tumours

Schroder, M., Queißer, W 376

Primary Gastrointestinal Lymphoma

Hünerbein, M., Schlag, P. M 384

Diagnosis and Surgical Treatment of Gastric Sarcoma

Lehnert, Th., Sinn, H.-P., Wolf, 0391

Treatment of Anal Cancer

Witzigmann, H, Hehl, A. J., Meyer, EM., Witte, J 398

Originalarbeiten 
Biologische Relevanz von Autoantikörpern gegen p53 im Serum von Patientinnen mit metastasiertem Mammakarzinom Porzsolt, E, Schmid, M., Höher, D., Muche, R., Gaus, W,

Montenarh, M

Einfluß von Alter und Operationsart auf die Lebensqualität von Mammakarzinompatientinnen Neises, M., Sabok Sir, M., Strittmatter, H.-X, Wischnik, A., Melchert, E

Ultraschallgeführte Feinnadelaspiration bei Malignomverdacht: Diagnostische Sicherheit, Bestimmung der Histogenese und Nutzen für den Patienten Schoengen, A., Binder, T., Schiffelholz, W., Schulz, P. C, Zeelen, U

B2-Mikroglobulin-Bestimmung im Serum zur Stadieneinteilung und Verlaufsbeobachtung multipler Myelome und weiterer Nicht-Hodgkin-Lymphome

Poley, S., Fateh-Mogtiadam, A., Nüssler, V., Pahl, H

Fax-Kommunikation

Neopterinausscheidung im Ijrin beim kolorektalen Karzinom

Melichar, B., Jandík, P., Solichová, D., Bures, X, Lukes, J.,

Mergancová, J., Voboril, Z

Original Articles

Biologic Relevance of Auto-Antibodies against p53 in Patients with Metastatic Breast Cancer Porzsolt, E, Schmid, M., Höher, D., Muche, R., Gaus, W.,

402 Montenarh, M 402

Influence of Age and of Different Operative Methods on the Quality of Life in Patients with Breast Cancer

Neises, M., Sabok Sir, M., Strittmatter, H.-J., Wischnik, A.,

410 Melchert, E 410

Fine-Needle Aspiration Guided by Ultrasound in Suspected Cancer: Diagnostic Accuracy, Determination of Histogenesis and Patient Benefit

Schoengen, A., Binder, T., Schiffelholz, W., Schulz, P. C,

420 Zeelen, U 420

Serum B2-Microglobulin for Staging and Monitoring of Multiple Myelomas and Other NonHodgkin Lymphomas

428 Poley, S., Fateh-Moghadam, A., Nüssler, V., Pahl, H 428

Fax Communication

Urinary Neopterin Excretion in Colorectal Cancer

Melichar, B., Jandík, P., Solichová, D., Bures, J., Lukes, X,

434 Mergancová, X, Voboril, Z 434

Fortsetzung aufSeite 344

Continued on page 344

Band 17, Heft 4, August 1994

International Journal for Cancer Research and Treatment

Inhalt

Contents 
Briefe an die Herausgeber

Aulitzky, W. E., Huber, Ch. zu:

Morant R, et at: Phase-II-Studie beim metastasierten Nierenzellkarzinom mit einer Kombination von rekombinantem Interferon-alpha $2 \mathrm{~b}$ und rekombinantem Interleukin-2

im Dauertropf 437

Funke I, et al: Subkutanes IL-2 und niedrigdosiertes IFN- $\alpha$ 2a in der Therapie unselektierter Patienten mit fortgeschrittenem Nierenzellkarzinom 437

Weh, H.-J. zu:

Schoengen A, et al: Ultraschallgefiihrte Feinnadelaspiration bei Malignomverdacht 437

Kayar, R. zu:

Obermair A, et al: Gefäßinvasion und Frührezidiv beim

Mammakarzinom: Vorläufige Ergebnisse 438

Letters to the Editors

Aulitzky, W. E., Huber, Ch. concerning:

Morant R, et al: Treatment of patients with metastatic renal cell carcinoma with subcutaneous recombinant interferonalpha $2 \mathrm{~b}$ and continuous infusion of recombinant interleukin-2: A phase II study $\quad 437$

Funke I, et al: Subcutaneous IL-2 and low-dose IFN- $\alpha$ 2a in the treatment of unselected patients with advanced renal cell cancer 437

Weh, H.-J. concerning:

Schoengen A, et al: Fine-needle aspiration guided by ultrasound in suspected cancer 437

Kayar, R. concerning:

Obermair A, et al: Vessel invasion predicts early recurrence in breast cancer: Preliminary results 438

Klinische Information

Metastasenchirurgie der Lunge im interdisziplinären Konzept

Schirren, J., Trainer, S., Krysa, S., Bülzebruck, H., Schneider, P., Drings, P., Vogt-Moykopf, 1439

Die Onkologievereinbarung

Kleeberg, U. R 449

Qualitätssicherung in der Onkologie: Definition der Behandlungsziele und Zielerreichung Porzsolt, E, Wirth, A., Mayer-Steinacker, R., Suhr, P., Warszawski, N., Schulte, M., Negri G., Kleeberger, L., Link, K.-H, Betz, E.,

Sarkar, M. R., Röttinger, E 454

Der 1995er Pezcoller-Preis fur Onkologie: 100000 E.C.U 458

Buchbesprechung $\quad 459$

Mitteilungen onkologischer Gesellschafien 460

Impressum 341

Inhaltsverzeichnis $\quad 342$ 
Hinweise für Autoren 463

Clinical Information

Surgical Treatment of Pulmonary Metastases in an Interdisciplinary Concept

Schirren, J., Trainer, S., Krysa, S., Bülzebruck, H., Schneider, P.,

Drings, P., Vogt-Moykopf, 1439

The Oncology Agreement

Kleeberg, U. R 449

Quality Assurance in Oncology: Definition of Treatment Goal and Assessment of Its

Achievement

Porzsolt, E, Wirth, A., Mayer-Steinacker, R., Suhr, P., Warszawski, N.,

Schulte, M., Negri G, Kleeberger, L., Link, K.-H., Betz, E.,

Sarkar, M.R., Röttinger, E 454

1995 Pezcoller Award for Oncology: 100,000 E.C.U 458

Book Review 459

Reports of Oncological Societies 460

Imprint $\quad 341$

Contents $\quad 342$

Instructions to Authors 463

Bibliographischer Hinweis: Inhaltsverzeichnisse dieser Zeitschrift erscheinen regelmäßig in current contents ${ }^{\circledR}$ sowie in anderen bibliographischen Diensten. 